\title{
MARKOV CHAIN MONTE CARLO SIMULATION MODEL FOR RISK ASSESSMENT THE POWER SYSTEMS FOR ELECTROMOBILITY USE
}

\begin{abstract}
A simulation model to evaluate risks in Power Systems including green energy sources to generate electricity for electro mobility use is presented in the paper. The model allows to calculate risk indicator that characterize the performance of the Power Systems. The model considers the additional risks of wind and solar variability in the Power Systems, through wind farms and PV farms, respectively. Also, in the recent years, the number of electric vehicles $(E V s)$ on the road have been rapidly increasing. Charging this increasing number of EVs is expected to have an impact on the power grid especially if high charging powers and opportunistic charging are used. Multiple papers have observed that the charging stations are used by multiple users during the day. In a context where electric mobility is gaining increasing importance as a more sustainable solution for urban environments, this work presents the optimization of charging profiles of the potential users of these charging stations. We analyzed the charging profiles in a power grid with renewables sources of energy and we determine the optimal charging profiles for the power grid based on maximizing the energy delivered by renewable sources of energy.
\end{abstract}

Keywords: Power system, Monte Carlo simulation, Markov chains 


\section{Introduction}

Cities play a key role in sustainable development. Urban growth must be managed in ways that support and drive economic development and achieve social cohesion and environmental sustainability. The concept of Smart Cities emerged in the same way as Smartphones or Smart TVs. Several initiatives are being developed as part of Smart City projects; however, there is a lack of consistent indicators, databases and methodologies for assessing, financing, and implementing these kinds of initiatives. Smart City projects today are classified according to six clusters known as axes: Mobility, Environment, Government, Economy, People and Living. The reference [4] show dynamically and graphically the scope of development of Spanish Smart City initiatives in terms of mobility and environmental issues, as two of the fundamental axes of Smart City development. The study was carried out in the 62 cities in the Spanish Smart Cities Network (RECI). The interactive map describes the status of Spanish cities by means of socioeconomic and demographic indicators and provides a thorough assessment of the maturity of Smart Cities based on their variables.

In the axes Mobility, one example is the reference [7] because show that a visualization and simulation of Intelligent Transportation Systems (ITS) for future city models is a key research area to bring better traffic safety and efficiency solutions in smart cities. However, the cost of deploying large-scale testbeds to analyze the performance of these solutions is prohibitively huge. Therefore, cooperative ITS simulation platforms are essential to test the performance of such solutions before their actual deployment. In order to fulfill this requirement, reference [7] developed PySNS3 (a Python based framework for bidirectional coupling between NS3 and SUMO). To test the robustness and reliability of proposed framework they have compared its mobility as well as communication related simulation results with state-of-the-art NS2-mobility-model. They have performed a simulation scenario of Harbin city that includes evaluation of 802.11p MAC/PHY characteristics, the architecture of Wireless Access in Vehicular Environment (WAVE), and prediction of the vehicular Edge computation capacity. They have also performed the evaluation of a traffic efficiency application using proposed framework to reduce the fuel consumption and waiting time. The simulation results proved that the proposed framework can offer dynamic coupling between SUMO and NS3 for the evaluation of Edge computing solutions of ITS for future city models.

The sustainable governance of transport systems remains a significant challenge for policy makers worldwide, particularly in cities. Urban areas are developing rapidly from a technological viewpoint, and innovative technologies create new possibilities for smart mobility management. For this reason, reference [8] investigates the relationship between the implementation of the smart city concept and the idea of sustainable transport, particularly regarding the reduction of transport generated $\mathrm{CO}_{2}$ emissions. The study estimates $\mathrm{CO}_{2}$ emissions for different potential scenarios of development for the Warsaw transport system until 2050 using the United Nations for FITS (For Future Inland Transport Systems) model. The study also analyses the additional impact on $\mathrm{CO}_{2}$ emissions of smart 
city elements as determinants of mobility. The results show that meeting the reduction targets set by the European Union 2011 White Paper on Transport will be challenging, requiring an in-depth transformation of the transport and energy sectors. This study also confirms that smart city solutions can play a crucial role in mitigating transport emissions and meeting reduction goals. The conclusions provide important insights for the design of smart mobility governance and enhance the relationship between transport policy and research.

One example of mitigation solution in the transport are electrical vehicles. With the recent advances in battery technology and the resulting decrease in the charging times, public charging stations are becoming a viable option for Electric Vehicle (EV) drivers. Concurrently, emergence and the wide-spread use of location-tracking devices in mobile phones and wearable devices has paved the way to track individual-level human movements to an unprecedented spatial and temporal grain. Motivated by these developments, reference [6] propose a novel methodology to perform data-driven optimization of EV charging station locations. They formulate the problem as a discrete optimization problem on a geographical grid, with the objective of covering the entire demand region while minimizing a measure of drivers' total excess driving distance to reach charging stations, the related energy overhead, and the number of charging stations. Since optimally solving the problem is computationally infeasible, they present computationally efficient solutions based on the genetic algorithm. They then apply the proposed methodology to optimize EV charging stations layout in the city of Boston, starting from Call Detail Records (CDR) of one million users over the span of 4 months. The results show that the genetic algorithm provides solutions that significantly reduce drivers' excess driving distance to charging stations, energy overhead, and the number of charging stations required compared to both a locally optimized feasible solution and the current charging station deployment in the Boston metro area. They further investigate the robustness of the proposed methodology and show that building upon well-known regularity of aggregate human mobility patterns, the layout computed for demands based on the single day movements preserves its advantage also in later days and months. When collectively considered, the results presented in this paper indicate the potential of data-driven approaches for optimally placing public charging facilities at urban scale.

In the recent years, the number of electric vehicles (EVs) on the road have been rapidly increasing. Charging this increasing number of EVs is expected to have an impact on the electricity grid especially if high charging powers and opportunistic charging are used. Several models have been proposed to quantify this impact. Multiple papers have observed that the charging stations are used by multiple users during the day. However, this observation was not assumed in any previous model. Moreover, none of the previous models relied on geospatial maps to extract information about the parking lots (where charging stations are installed) and the charging profiles of the potential users of these charging stations. In the reference [5], a spatial Markov chain model is developed to model the charging load of EVs in cities. The model assumes three distinct charging profiles: Work, Home, and Other. Geospatial maps were used to estimate the charging 
profile, or mixture of profiles, of the charging stations based on the nearby building types. Also, the proposed model can potentially be used along with renewable energy sources models to estimate the spatial-temporal synergy potentials between the two technologies. Evaluating the synergy potentials might be of value to grid operators, policy makers, market traders, etc.

In the contest of European and National choices regarding energy and mobility policies, the reference [1] introduce reasonable guidelines for the local promotion of electric mobility in an area of central Italy and possibly to extend the methodology to other regions/countries. In fact, the path towards car electrification is started worldwide, but the rate of increase of electrically powered cars will depend primarily on charging stations availability. In this respect, utilities that manage/own local grids may be key actors by investing on infrastructures and thus locally creating the conditions for the spreading of electric cars. A techno-economic analysis of the investment of a local utility has been performed considering medium- and long term-forecasts of electricity and fuel prices, as well as electric cars market share and economic risk. The study proved that an investment in urban charging station infrastructure in Italy can be profitable even without incentives, with a payback period of 4-5 years. The results of the analysis have been exploited by an Italian utility which owns/manages the local electric grid in two municipalities. The first steps of the plan implementation, which started in 2014, are reported in the paper.

In a context where electric mobility is gaining increasing importance as a more sustainable solution for urban environments, the reference [2] presents an analysis of electric mobility feasibility and adequacy based on private users' naturalistic driving data. Several scenarios were tested to evaluate different charging event opportunities and their impacts on electric mobility feasibility. In more detail, scenario 1 considered that vehicles would recharge whenever they are stopped for 2, 4 or $6 \mathrm{~h}$, either on weekdays or weekend days; scenario 2 tested the hypothesis of recharging only during the night period; and scenario 3 assumed that vehicles would recharge during the day on weekdays. Furthermore, the potential energy impacts of electric mobility at a city level, by applying a driver and street level approach, were also estimated.

Photovoltaic generation and electric mobility are disruptive technologies in the power and transport sectors, and both pose challenges for power grids. The scholarship has focused on potential synergies when these two technologies are combined, and recent research shows that interactions between photovoltaic generation and electric mobility could decrease the overall burden on power grids and empower one technology with the other's specificities. Electric vehicles (EV) could use photovoltaic energy (PV) and benefit from cheap carbon-free electricity for charging. In return, PV systems could use the bidirectional flexibility of EV batteries to maximize their self-consumption. As these synergies operate, economic spillovers from these technologies are expected to improve, thus further leveraging their joint deployment. The reference [3] develop a systematic framework to review the various underlying conditions for synergy as they have been studied in the literature. It emerges that this synergy is driven by technical as well as economic factors. First, it happens in the mid-scale spatial configuration (large workplace buildings and 
charging stations) and less obviously at other scales (eg. households, territories) and in technologically diversified systems. Second, although under-studied in the literature, the economic context (level of cooperation between stakeholders, regulation and policy, etc.) of interactions between PV and EV is crucial for successful synergy. Finally, they identify several remaining issues with these conditions that warrant further research.

\subsection{Contribution of the paper}

Based on the previous references, we propose a new approach related with several topics defined above. From the reference [4], we adapt the Smart City concepts in the axes mobility, and we connected with the reference [7] because we contribute with the idea that a cooperative Intelligent Transportation Systems (ITS) simulation platforms are essential to test the performance of such solutions before their actual deployment. From the reference [8] we assume the relationship between the implementation of the smart city concept and the idea of sustainable transport, particularly regarding the reduction of transport generated $\mathrm{CO}_{2}$ emissions, in this case using V2G system, and also we contribute with the proposal of the reference [6] because we relate the novel methodology to perform data-driven optimization of EV charging station locations of the reference [6] with the reference [5] that assumes three distinct charging profiles: Work, Home, and Other. In the end, we propose analyze the impact of V2G system in the grid motivated for the reference [2] that show several scenarios to evaluate different charging event opportunities and their impacts on electric mobility feasibility, and for the reference [3], this last reference show how photovoltaic generation and electric mobility are disruptive technologies in the power and transport sectors, and both pose challenges for power grids. The paper summarizes all these ideas in a new approach.

\section{Material and methods}

\subsection{Description}

The microgrid is divided into four important parts: A diesel generator, acting as the base power generator; a PV farm combined with a wind farm, to produce renewable energy; a V2G system installed next to the last part of the system which is the load of the grid. The size of the microgrid represents approximately a community of a thousand households during a low consumption day in spring or fall. There are 100 electric vehicles in the base model which means that there is a 1:10 ratio between the cars and the households. This is a possible scenario in a foreseeable future (see fig. 1). 


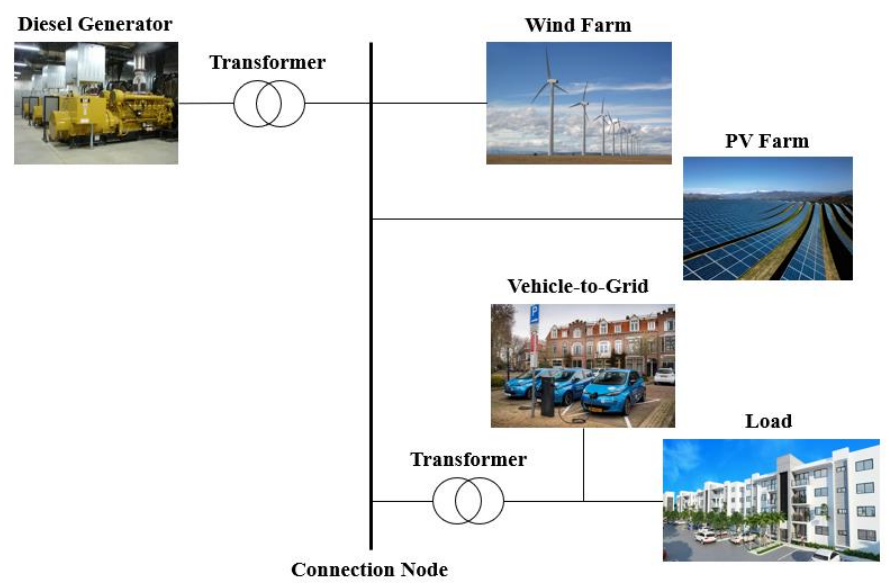

Fig. 1. Microgrid diagram

\subsection{Diesel Generator}

The diesel generator balances the power consumed and the power produced, acting as the base power generation. The model proposed to simulate generating capacity of the diesel generator is defined below:

$$
G_{i}(t)=\left\{C_{i} \text { if } t_{\text {initial }} \leq t<t_{\text {final }}\right.
$$

where $C_{i}$ nominal capacity of the diesel generator $i$; $t$ final - $t$ initial is the simulation window, usually 24 hours and $i=1,2, \ldots, N G$ (number of diesel generators).

\subsection{Renewable Energy}

There are two sources of renewable energy in this microgrid. First, a PV farm produces energy proportional to three factors: the size of the area covered by the PV farm, the efficiency of the solar panels and the irradiance data. Second, a wind farm model produces electrical power following a non-linear relationship with the wind. When the wind reaches a nominal value, the wind farm produces the nominal power. The wind farm trips from the grid when the wind speed exceeds the maximum wind value, until the wind gets back to its nominal value.

Wind Farm modeling. The generation capacity of the wind farm $G_{W F}(t)$ at the instant of time $t$ is determined by $P W T_{i}$ of each wind turbine $i$. In this case, the power $P W T_{i}$ depends on the wind $S W t$. In the proposed model we use Weibull simulation approaches for the wind speed, so Weibull model generates random values from the density function adjusted with 
historical values. In the case of the power delivered by each wind turbine we use the nonlinear relationship between wind speed and wind turbine power.

The wind speed is simulated with the Weibull model estimating approximately the shape and scale parameters of the probability distribution density function with $\mu_{s w}$ and $\sigma_{s w}$. The Weibull probability distribution function for wind speed $v$ is denoted as $f(v), \beta$ and $\delta$ are the shape and scale parameters of the distribution function respectively.

$$
f(v)=\frac{\beta}{\delta}\left(\frac{v}{\delta}\right)^{\beta-1} e^{\left[-\left(\frac{v}{\delta}\right)^{\beta}\right]}
$$

The parameters $\beta$ and $\delta$ are obtained with the following expressions:

$$
\begin{aligned}
& \beta=\left(\frac{\sigma_{s w}}{\mu_{s w}}\right)^{-1.086} \\
& \delta=\frac{\mu_{s w}}{\Gamma(1+1 / \beta)}
\end{aligned}
$$

The inverse of cumulative probability distribution function (5) allows us to simulate the wind speed generating $u$ uniformly distributed random numbers $[0,1]$ as shown in (6).

$$
\begin{gathered}
F(v)=1-e^{\left[-\left(\frac{v}{\delta}\right)^{\beta}\right]} \\
S W_{t}=v=-\delta \ln (1-u)^{1 / \beta}=-\delta \ln (u)^{1 / \beta}
\end{gathered}
$$

The power delivered by each wind turbine is estimated with the function (7). The nonlinear relationship between wind speed and wind turbine power is given by,

$$
P W T\left(S W_{t}\right)=\left\{\begin{array}{ccc}
0 & \text { if } & 0 \leq S W_{t}<V_{c i} \\
\left(A+B \times\left(S W_{t}\right)+C \times\left(S W_{t}\right)^{2}\right) \times P_{r} & \text { if } & V_{c i} \leq S W_{t}<V_{r} \\
P_{r} & \text { if } & V_{r} \leq S W_{t}<V_{c o} \\
0 & \text { if } & S W_{t} \geq V_{c o}
\end{array}\right.
$$

where $P_{r}, V_{c i}, V_{r}$ and $V_{c o}$ are nominal output power, wind speed necessary for start-up, wind speed corresponding to the nominal power of the wind turbine and cutting wind speed per wind turbine safety reasons respectively.

The constants $A, B$, and $C$ depend on $V_{c i}, V_{r}$ and $V_{c o}$ as shown in (8). 


$$
\begin{aligned}
& A=\frac{1}{\left(V_{c i}-V_{r}\right)^{2}}\left\{V_{c i}\left(V_{c i}+V_{r}\right)-4 V_{c i} V_{r}\left[\frac{V_{c i}+V_{r}}{2 V_{r}}\right]^{3}\right\} \\
& B=\frac{1}{\left(V_{c i}-V_{r}\right)^{2}}\left\{4\left(V_{c i}+V_{r}\right)\left[\frac{V_{c i}+V_{r}}{2 V_{r}}\right]^{3}-\left(3 V_{c i}+V_{r}\right)\right\} \\
& C=\frac{1}{\left(V_{c i}-V_{r}\right)^{2}}\left\{2-4\left[\frac{V_{c i}+V_{r}}{2 V_{r}}\right]^{3}\right\}
\end{aligned}
$$

The delivered power of the wind farm is defined below as:

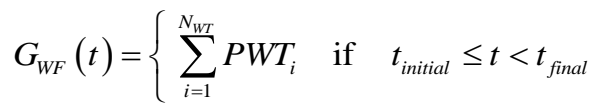

where $i=1,2, \ldots, N_{W T}$.

Photovoltaic solar farm modeling. The generation capacity $G_{P V}(\mathrm{t})$ of a photovoltaic solar farm at instant of time $t$ is determined by $P P V_{i}$ of each photovoltaic panel $i$ in the solar farm. In this case, the solar power $P P V_{i}$, depends of solar radiation $S R_{i}(t)$.

The literature shows a wide diversity of methods and models to simulate the behavior of $S R_{i}(t)$, in this paper the behavior of solar radiation is assumed as a known variable. The power output of a photovoltaic solar farm is proportional to the solar radiation in the area where it is located, and it is usual that there are weather stations in the places where the photovoltaic solar farm are to be installed, for this reason it is also assumed in the paper that the power output $G_{P V}(t)$ is a known variable. The photovoltaic solar farm has a typical configuration shown in fig. 2. In this paper it is assumed that each Array is independent.

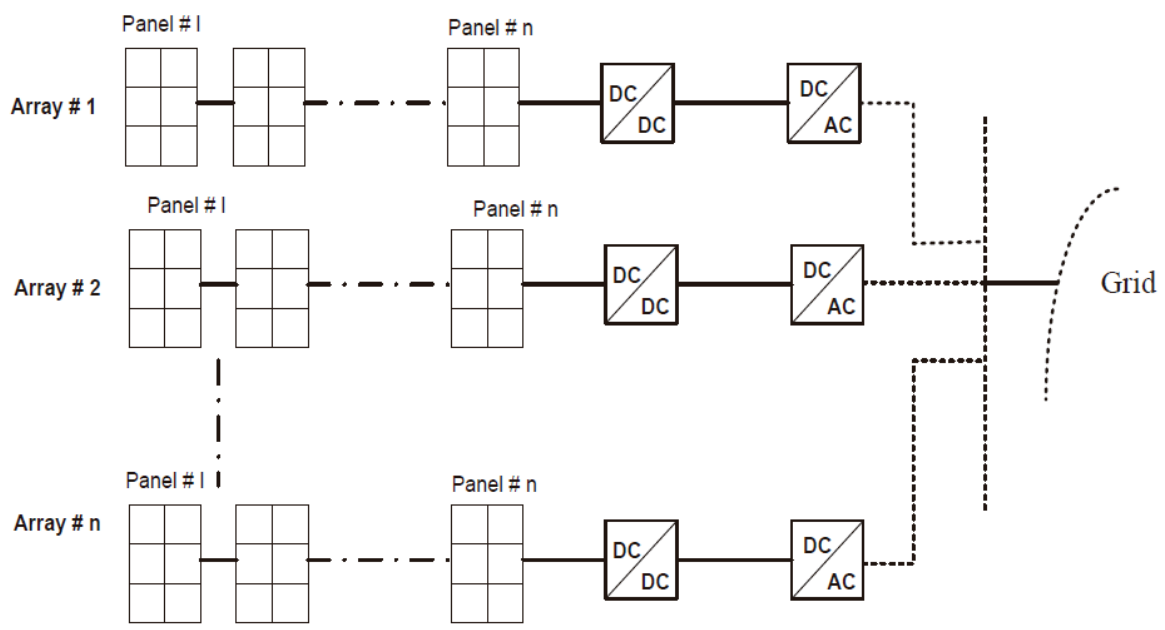

Fig. 2. Typical configuration of a photovoltaic solar farm 
When the $P P V_{i}$ power delivered by each array $i$ is known, the $G_{P V}(t)$ power delivered for the photovoltaic solar farm is defined as:

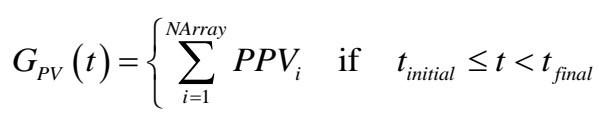

where $i=1,2, \ldots, N_{\text {Array }}$ (number of arrays in the photovoltaic solar farm).

\subsection{Vehicle-to-Grid}

The V2G has the function of controls the charge of the batteries connected to it. We can find five typical car-user profiles:

Profile \#1: People going to work with a possibility to charge their car at work.

Profile \#2: People going to work with a possibility to charge their car at work but with a longer ride.

Profile \#3: People going to work with no possibility to charge their car at work.

Profile \#4: People staying at home.

Profile \#5: People working on a night shift.

The State of Charge (SOC) of the battery is necessary to know because with this information we can determine the best car-user profile for the grid. To simulate the battery behavior, a circuit was implemented that allows estimating the behavior of: SOC (\%), temperature in the battery (Celsius degrees), current (Ampere) and voltage (Volt).

In this paper, the battery model used relate the voltage according to the charge with the following relationship,

$$
V=V_{0}\left(\frac{S O C}{1-\beta(1-S O C)}\right)
$$

where SOC (state-of-charge) is the ratio of current charge to rated battery capacity; $V_{0}$ is the voltage when the battery is fully charged at no load, as defined by the Nominal voltage parameter; $\beta$ is a constant that is calculated, so the battery voltage is $V_{1}$ when the charge is $A H_{1}$.

The equation defines an approximate relationship between voltage and remaining charge. This approximation replicates the increasing rate of voltage drop at low charge values and ensures that the battery voltage becomes zero when the charge level is zero. The advantage of this model is that it requires few parameters, which are readily available on most datasheets.

In the paper, the battery model used provide additional parameters to define battery behavior according to the temperature. The extended equations for the voltage when the thermal port is exposed are, 


$$
\begin{aligned}
& V=V_{0 T}\left(\frac{S O C}{1-\beta(1-S O C)}\right) \\
& V_{0 T}=V_{0}\left(1+\lambda_{V}\left(T-T_{1}\right)\right)
\end{aligned}
$$

where $T$ is the battery temperature; $T_{1}$ is the nominal measurement temperature; $\lambda_{V}$ is the parameter temperature dependence coefficient for $V_{0}$ and $\beta$ is calculated in the same way before, using the temperature-modified nominal voltage $V_{0 T}$.

The internal series resistance, self-discharge resistance, and any charge-dynamic resistances are also functioning of temperature:

$$
R_{T}=R\left(1+\lambda_{R}\left(T-T_{1}\right)\right)
$$

where $\lambda_{R}$ is the parameter temperature dependence coefficient.

All the temperature dependence coefficients are determined from the corresponding values you provide at the nominal and second measurement temperatures. If it is including charge dynamics in the model, the time constants vary with temperature in the same way.

The battery temperature is determined from a summation of all the ohmic losses included in the model:

$$
M_{t h} \dot{T}=\sum_{i} \frac{V_{T, i}^{2}}{R_{T, i}}
$$

where $M_{t h}$ is the battery thermal mass; $i$ corresponds to the $i$ th ohmic loss contributor; The losses include: Series resistance, Self-discharge resistance, First charge dynamics segment, Second charge dynamics segment, $V_{T, i}$ is the voltage drop across resistor $i$ and $R_{T, i}$ is resistor $i$.

\subsection{Load}

The load is composed of residential load or/and industrial load (see fig. 3). The residential load and industrial load follow a consumption profile with a given power factor. 


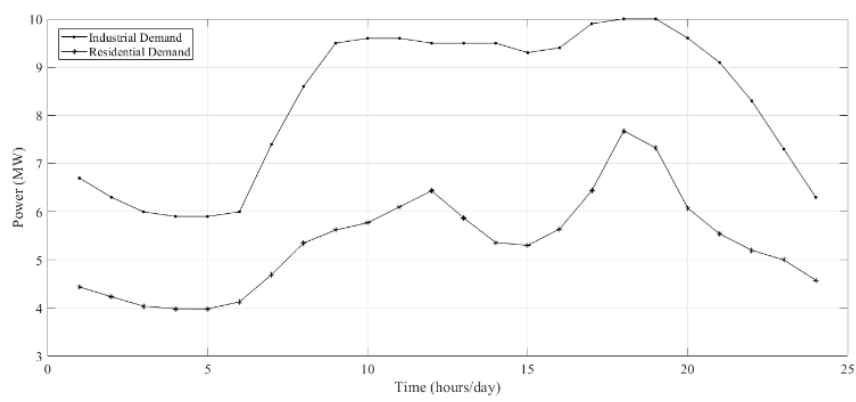

Fig. 3. System load

The load curve model is usually represented by the Daily Peak Load Variation Curve (DPLVC) or the Hourly Load Duration Curve (LDC). We used LDC because integrates the load curve chronological behavior.

\subsection{Scenario}

The simulation lasts 24 hours. The solar intensity follows a normal distribution where the highest intensity is reached at midday. The wind varies greatly during the day and has multiple peaks and lows. The residential load and industrial load follow a typical pattern like a normal household consumption. The consumption is low during the day and increases to a peak during the evening, and slowly decreases during the night.

\subsection{Simulation}

In this paper, we consider a diesel generator with generating capacity $C_{1}=15 \mathrm{MW}$ (acting as the base power generator all day, 24 hours).

The PV farm have $G_{P V}=8 \mathrm{MW}$ peak generating capacity and when we consider the size of the area covered by the PV farm, the efficiency of the solar panels and the irradiance data we obtain fig. 4. We used the numerical vector (see fig. 4) to simulate the capacity behavior of the PV farm during the day.

In the case of the wind farm, we calculate the shape and scale parameters of the Weibull probability distribution density function to simulate the wind, assuming $\mu_{S W}=5.4 \mathrm{~m} / \mathrm{s}$ and $\sigma_{S W}=2.3$. The wind turbine used in this paper has a nominal output power $P_{r}=4.5 \mathrm{MW}$, the cut-in speed wind $V_{c i}=4 \mathrm{~m} / \mathrm{s}$, the rated speed wind $V_{r}=10 \mathrm{~m} / \mathrm{s}$ and the cut-out wind speed $V_{c o}$ is $25 \mathrm{~m} / \mathrm{s}$. Figure 5 show the capacity behavior of the wind farm. 


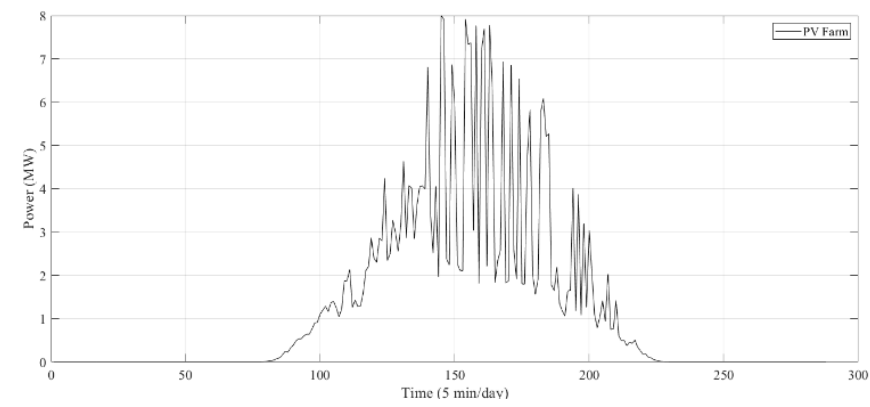

Fig. 4. Solar power

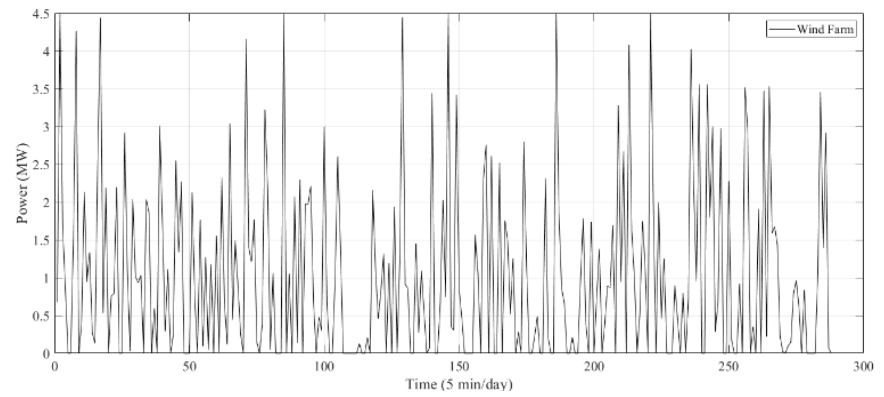

Fig. 5. Wind power

The V2G system installed next to the last part of the system which is the load of the grid have the capacity of $4 \mathrm{MW}$. There are 100 electric vehicles and each car have the capacity of $40 \mathrm{~kW}$. In this paper, we present the optimization of charging profiles of the potential users of these charging stations. We analyzed the charging profiles in a power grid with renewables sources of energy and we determine the optimal charging profiles for the power grid based on maximizing the energy delivered by renewable sources of energy during the day. In fact, we consider that V2G system as a load, and based in this, we assuming the below mathematical model for controlled loads (V2G):

$$
P_{V 2 G_{j}}(t)=\left\{\begin{array}{c}
P_{j} \times S O C_{j} \text { when } t_{s o_{j}}<t<t_{s o_{j}}+t_{o t_{j}} \\
0 \text { otherwise } \forall j
\end{array}\right.
$$

where $S O C_{j}$ is the State of Charge of the electrical car $j$ at the time instant $t, P_{j}$ is the power consumption of the electrical car $j, t_{s o j}$ is the start time of consumption of the electrical car $j, t_{o t j}$ is the operating consumption time of the electrical car $j$.

Based on the assumption that we can control the load (V2G) with a smart grid, we propose an optimization model to determine the optimal charging profiles maximizing the 
energy delivered by renewable sources of energy during the day. The objective function is defined as:

$$
\begin{aligned}
& \min \sqrt{\frac{1}{N} \sum_{t=1}^{N}\left[G_{i}(t)+G_{W F}(t)+G_{P V}(t)-\left(\sum_{j=1}^{N_{V 2 G}} P_{V 2 G_{j}}(t)+D(t)\right)\right]^{2}} \\
& \text { for } t=1,2, \ldots, N \\
& \text { subject to: } G_{i}(t)+G_{W F}(t)+G_{P V}(t) \geq \sum_{j=1}^{N_{V 2 G}} P_{V 2 G_{j}}(t)+D(t) \quad \forall t
\end{aligned}
$$

where $N$ is the samples number of the simulation window and $D(t)$ is the residential and/or industrial load of the system.

In this paper, we focus the attention in the validation of the proposal model. The objective function is non-linear stochastics function, for this reason, we use GA and PSO algorithmic for the problem solution. In this scenario, the V2G system fleet has 100 electrical car and each one has $40 \mathrm{~kW}$ of capacity. For the validation of the model, we assuming a scenario (no real) where every car need charge the battery for 8 hours to complete the full charge, are available to charge during all day and the smart grid can control the best moment to charge the battery of each car.

Figure 6 show the convergence process of both algorithms. PSO algorithm (local search) in few iterations find a local minimum with a good solution for this problem. On another hands, GA algorithm (global search) needs more iterations, find betters solutions that PSO algorithm, but the difference is not significative.

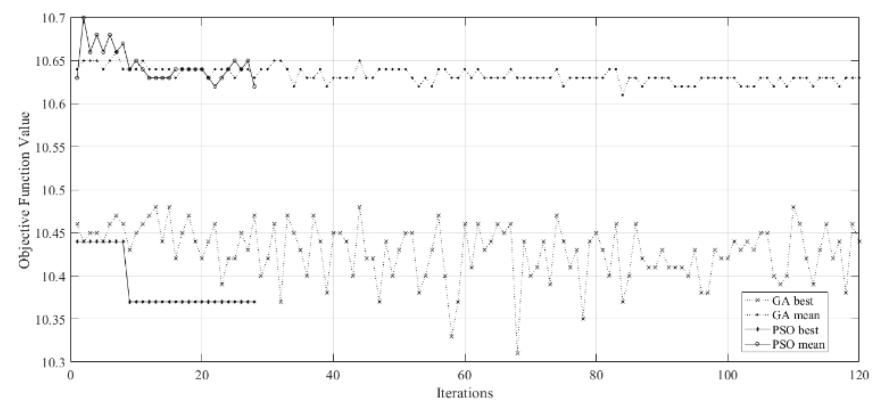

Fig. 6. Convergence process

\section{Conclusions}

In the recent years, the number of electric vehicles (EVs) on the road have been rapidly increasing. Charging this increasing number of EVs is expected to have an impact on the 
power grid especially if high charging powers and opportunistic charging are used. Multiple papers have observed that the charging stations are used by multiple users during the day. In a context where electric mobility is gaining increasing importance as a more sustainable solution for urban environments, this work presents the optimization of charging profiles of the potential users of these charging stations. We analyzed the charging profiles in a power grid with renewables sources of energy and we determine the optimal charging profiles for the power grid based on maximizing the energy delivered by renewable sources of energy.

\section{Acknowledgement}

The work has been financially supported by the Polish Ministry of Science and Higher Education.

\section{References}

1. Comodi G., Caresana F., Salvi D., Pelagalli L., Lorenzetti M.: Local promotion of electric mobility in cities: Guidelines and real application case in Italy. Energy, Vol. 95, 2016.

2. Faria M., Duarte G., Baptista P.: Assessing electric mobility feasibility based on naturalistic driving data. Journal of Cleaner Production, Vol. 206, 2019.

3. Hoarau Q., Perez Y.: Interactions between electric mobility and photovoltaic generation: A review. Renewable and Sustainable Energy Reviews, Vol. 94, 2018.

4. Neus Baucells Aletà, Concepción Moreno Alonso and Rosa M. Arce Ruiz. Smart Mobility and Smart Environment in the Spanish cities. 3rd Conference on Sustainable Urban Mobility, 3rd CSUM 2016, 26-27 May 2016, Volos, Greece. Transportation Research Procedia, Vol. 24, 2017.

5. Shepero M., Munkhammar J.: Spatial Markov chain model for electric vehicle charging in cities using geographical information system (GIS) data. Applied Energy, Vol. 231, 2018.

6. Vazifeh M.M., Zhang H., Santi P., Ratti C.: Optimizing the deployment of electric vehicle charging stations using pervasive mobility data. Transportation Research Part A, Vol. 121, 2019.

7. Wang T., Hussain A., Bhutta M.N.M., Cao Y.: Enabling bidirectional traffic mobility for ITS simulation in smart city environments. Future Generation Computer Systems, Vol. 92, 2019.

8. Zawieska J., Pieriegud J.: Smart city as a tool for sustainable mobility and transport decarbonization. Transport Policy, Vol. 63, 2018. 